\title{
Enhancing the Violation of the Einstein-Podolsky-Rosen Local Realism by Quantum Hyper-entanglement
}

\author{
Marco Barbieri ${ }^{1}$, Francesco De Martini ${ }^{1}$, Paolo Mataloni ${ }^{1}$, Giuseppe Vallone ${ }^{2}$, Adán Cabello ${ }^{3}$ \\ ${ }^{1}$ Dipartimento di Fisica dell'Università "La Sapienza" and \\ Consorzio Nazionale Interuniversitario per le Scienze Fisiche della Materia, \\ 00185 Roma, Italy \\ ${ }^{2}$ Dipartimento di Fisica Teorica Università di Torino and \\ Istituto Nazionale di Fisica Nucleare - sezione di Torino - 10125 Torino, Italy \\ ${ }^{3}$ Departamento de Física Aplicada II, \\ Universidad de Sevilla, 41012 Sevilla, Spain
}

\begin{abstract}
Mermin's observation [Phys. Rev. Lett. 65, 1838 (1990)] that the magnitude of the violation of local realism, defined as the ratio between the quantum prediction and the classical bound, can grow exponentially with the size of the system is demonstrated using two-photon hyper-entangled states entangled in polarization and path degrees of freedom, and local measurements of polarization and path simultaneously.

PACS numbers: 03.65.Ud, 03.67.Pp, 42.50.-p
\end{abstract}

Einstein, Podolsky, and Rosen (EPR) [1] believed that the results of experiments on a system $A$ which can be remotely predicted from the results of spacelike separated experiments on a system $B$ were determined only by $A$ 's local properties. Bell's discovery that this is not the case 2] meant a spectacular departure from the local realistic view of the world.

Not so long ago, it was thought that the magnitude of the violation of local realism, defined as the ratio between the quantum prediction and the classical bound, decreases as the size (i.e., number $n$ of particles and/or the number $N$ of internal degrees of freedom) grows, as a manifestation of some intrinsic aspect of the transition from quantum to classical behavior [3]. Later, it was found that this magnitude can remain constant as $N$ increases [4, 5, 6]. This constant behavior occurs in most bipartite $N$-level Bell inequalities [7]. However, there is some evidence that bipartite three-level systems can provide stronger violations of local realism than bipartite two-level systems [8].

It was Mermin [9] who showed that the ratio between the quantum prediction and the classical bound can grow as $2^{(n-1) / 2}$ in the case of $n$-qubit systems prepared in Greenberger-Horne-Zeilinger (GHZ) states 10]. However, this effect is difficult to observe in real experiments because it is difficult to produce GHZ states with $n>4$ 11, 12, 13, 14] and because $n$-particle GHZ states suffer a decoherence that also grows with $n$ 15].

The aim of this Letter is to describe an experiment in which Mermin's growing-with-size quantum nonlocality effect is observed. The experiment is based on two ingredients. On one hand, on a Bell inequality derived but from the EPR criterion for elements of reality [1] applied to higher dimensional local subsystems, where two compatible observables of the same particle can be regarded as simultaneous EPR elements of reality if the result of measuring each of them can be remotely pre- dicted with certainty and this prediction is independent of which other compatible observables are measured simultaneously. Examples of this type of Bell inequalities can be found in 16, 17, 18, 19]. Some of them have been recently tested in real experiments 20, 21]. A detailed discussion of these Bell inequalities based on the EPR elements of reality can be found in 22]. The second key ingredient is hyper-entanglement (i.e., entanglement involving different degrees of freedom [23, 24]), and particularly the possibility of producing double [20, 21, 25] (and, eventually, triple 26] ) Bell hyper-entangled states. Under some assumptions, hyper-entanglement allows us to replace $n$ two-level systems by two $N$-level systems, which significatively reduces the decoherence problems, simplifies the task of achieving space-like separation between measurements, and dramatically increases the efficiency of detecting EPR elements of reality (since each photodetection reveals $\log _{2} N$ elements of reality) 22]. While previous tests of Bell inequalities using hyperentangled states have confirmed entanglement in each of the degrees of freedom separately by using different local setups for each degree of freedom [25, 26], the local measurements in our experiment are designed to show entanglement in all degrees of freedom using the same setup.

Our experiment is based on the properties of the twophoton hyper-entangled state exhibiting entanglement both in polarization and momentum $\mathbf{k}$ degrees of freedom,

$$
|\Psi\rangle=\frac{1}{2}\left(|H\rangle_{u}|H\rangle_{d}-|V\rangle_{u}|V\rangle_{d}\right) \otimes\left(|l\rangle_{u}|r\rangle_{d}+|r\rangle_{u}|l\rangle_{d}\right),
$$

where $|H\rangle_{j}$ and $|V\rangle_{j}$ represent horizontal and vertical polarization, and $|l\rangle_{j}$ and $|r\rangle_{j}$ denote two orthonormal path states, i.e. $\mathbf{k}$-modes, for photon- $j(j=u, d)$. In the above expression, $l(r)$ and $u(d)$ correspond to the left (right) and up (down) sides of the entanglement ring 
(e-ring) of the parametric source (see Fig. 1a).

In the first part of the experiment we demonstrate that the state (11) violates two Bell inequalities, for polarization and path, separately (a similar experiment has been performed recently both by us and the Urbana group 25, 26]).

For this purpose, we consider the Clauser-HorneShimony-Holt (CHSH) 27] Bell operator for polarization $(\pi)$ observables

$$
\beta_{\pi}=-A_{\pi} \otimes B_{\pi}+A_{\pi} \otimes b_{\pi}+a_{\pi} \otimes B_{\pi}+a_{\pi} \otimes b_{\pi}
$$

where the incompatible polarization observables of the first and second photon are, respectively:

$$
\begin{aligned}
& A_{\pi}=|H\rangle\langle H|-| V\rangle\left\langle V\left|, \quad a_{\pi}=\right| V\right\rangle\langle H|+| H\rangle\langle V|, \\
& B_{\pi}=\frac{1}{\sqrt{2}}[|H\rangle\langle H|-| V\rangle\langle V|+| V\rangle\langle H|+| H\rangle\langle V|], \\
& b_{\pi}=\frac{1}{\sqrt{2}}[|V\rangle\langle V|-| H\rangle\langle H|+| V\rangle\langle H|+| H\rangle\langle V|],
\end{aligned}
$$

They are chosen to provide a maximum violation (i.e., $2 \sqrt{2}$ ) of the CHSH-Bell inequality $\left|\beta_{\pi}\right| \leq 2$. In addition, we consider the CHSH-Bell operator for path (k) observables

$$
\beta_{\mathbf{k}}=A_{\mathbf{k}} \otimes B_{\mathbf{k}}-A_{\mathbf{k}} \otimes b_{\mathbf{k}}+a_{\mathbf{k}} \otimes B_{\mathbf{k}}+a_{\mathbf{k}} \otimes b_{\mathbf{k}}
$$

where the (incompatible) path observables of the first and second photon are, respectively:

$$
\begin{aligned}
A_{\mathbf{k}} & =|l\rangle\langle r|+| r\rangle\langle l| \quad, \quad a_{\mathbf{k}}=i(|r\rangle\langle l|-| l\rangle\langle r|), \\
B_{\mathbf{k}} & =[(i+1)|r\rangle\langle l|-(i-1)| l\rangle\langle r|] / \sqrt{2}, \\
b_{\mathbf{k}} & =[(i-1)|r\rangle\langle l|-(i+1)| l\rangle\langle r|] / \sqrt{2},
\end{aligned}
$$

They are chosen to provide a maximum violation (i.e., $2 \sqrt{2}$ ) of the CHSH-Bell inequality $\left|\beta_{\mathbf{k}}\right| \leq 2$.

In the second part of the experiment, we demonstrate that the violation of the Bell inequalities grows exponentially with the number of internal degrees of freedom. On this purpose, we consider the $\pi-\mathbf{k}$ Bell operator

$$
\beta=\beta_{\pi} \otimes \beta_{\mathbf{k}},
$$

which requires measuring 4 alternative 4 -outcome local observables on each photon: $A_{\pi} A_{\mathbf{k}}, A_{\pi} a_{\mathbf{k}}, a_{\pi} A_{\mathbf{k}}$, and $a_{\pi} a_{\mathbf{k}}$ on the first photon, and $B_{\pi} B_{\mathbf{k}}, B_{\pi} b_{\mathbf{k}}, b_{\pi} B_{\mathbf{k}}$, and $b_{\pi} b_{\mathbf{k}}$ on the second photon (i.e., we have 16 different experimental configurations). Each of the local observables is the product of a polarization observable and a path observable. Any theory admitting EPR elements of reality must satisfy the following inequality

$$
|\beta| \leq 4,
$$

while quantum mechanics predicts a value of 8 (with ideal equipment). As a consequence, quantum nonlocality is expected to grow exponentially with the number $N$ of degrees of freedom, i.e. in our case the ratio between the quantum prediction and the classical bound grows as $2^{\frac{N}{2}}$. The Bell inequality (11) is based on Aravind's observation [17] that Mermin's growing-with-size quantum nonlocality effect also exists for $n / 2$ two-level Bell states. Under some assumptions [22], by hyper-entanglement we can replace $\mathrm{n}$ separated qubits by two N-level systems.

Before observing the quantum violation of the twodegree of freedom Bell inequality (7), we should test whether the assumptions leading to (7) are satisfied in our experiment or not. These assumptions are:

(i) The results of the measurements of each of the polarization and path observables on photon $u(d)$ can be predicted with near certainty (i.e., with a sufficiently large probability) from the results of remote measurements on photon $d(u)$ 28].

(ii) If the same element of reality (for instance $A_{\pi}$ ) appears in two different setups (for instance, $A_{\pi} A_{\mathbf{k}}$ and $A_{\pi} a_{\mathbf{k}}$ ), the remote prediction for $A_{\pi}$ must be the same in both setups [28].

To sum up, we must check that $A_{\pi}$ can be predicted (with almost perfect certainty) from different experiments on $B$, and that this prediction must not depend on whether $A_{\pi}$ is measured using, for instance, the setup $A_{\pi} A_{\mathbf{k}}$ or the setup $A_{\pi} a_{\mathbf{k}}$. This test requires measuring all possible combinations of product local observables sharing one polarization (or path) observable. For instance, the prediction for the value $A_{\pi}$ on the first photon must be the same, regardless of whether we measure $A_{\pi} A_{\mathrm{k}}$, or $A_{\pi} a_{\mathbf{k}}$, or $A_{\pi} B_{\mathbf{k}}$, or $A_{\pi} b_{\mathbf{k}}$ on the second photon, and regardless of whether we chose the setup $A_{\pi} A_{\mathbf{k}}$, or $A_{\pi} a_{\mathbf{k}}$, or $A_{\pi} B_{\mathbf{k}}$, or $A_{\pi} b_{\mathbf{k}}$ to measure $A_{\pi}$ on the first photon (and so on). If $(i)$ and (ii) are satisfied, then we can proceed with the second part of the experiment and look for violations of the two-degree of freedom Bell inequality (7), in agreement with the quantum prediction.

The whole experiment admit two different analysis. If we relax the EPR criterion and define elements of reality as those that can be predicted with almost perfect certainty, as in 28], then the inequality (7) is valid for all prepared pairs, and the experimental value of $\beta$ can be compared with the classical bound. However, if we use the original EPR criterion, then the inequality (7) is legitimate only for a fraction of pairs. Then, we should modify the classical bound of the inequality in order to take into account the effect of the fraction of the pairs for which the inequality is not valid 22]. In both approaches, a good measure of nonlocality is the ratio between the experimental value of $\beta$ and the maximal possible value allowed by the local realistic theories. This measure is related both to the number of bits needed to communicate nonlocally in order to emulate the experimental results by a local realistic theory, and also to the minimum detection efficiency needed for a loophole-free experiment. In this sense, a higher value of this ratio is a significant 


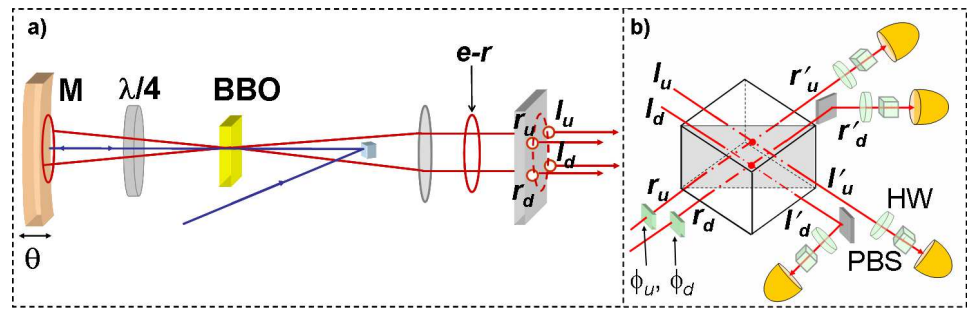

FIG. 1: a) Parametric source of polarization-momentum hyper-entangled two-photon states. The zero-order $\lambda / 4$ waveplate placed between $M$ and the BBO crystal, intercepts twice both back-reflected $\lambda$ and $\lambda_{p}$ beams and rotates by $\pi / 2$ the polarization of the back-reflected field at $\lambda$ while leaving the polarization state of the UV pump beam virtually undisturbed. The orthogonal section of the emission cone identifies the $e n$ tanglement ring $(e-r i n g)$. Phase setting $\theta=0, \pi$ are obtained by micrometric translation of $M$; b) Spatial coupling of input mode sets $l_{u}-l_{d}$ and $r_{u}-r_{d}$ on the $B S$ plane. The $B S$ output modes, $l_{u}^{\prime}, l_{d}^{\prime}, r_{u}^{\prime}$, and $r_{d}^{\prime}$ are analyzed each by a half-wave plate $(H W)$ and a polarization beamsplitter $(P B S)$. Glass plates on modes $r_{u}$ and $r_{d}$ are used in order to measure momentum observables.

step towards a loophole-free Bell test 22.

The source of entangled photons used in the experiment consists of a thin type I $\beta$-barium-borate (BBO) crystal slab operating under the double (back and forth) excitation of a cw $A r^{+}$laser $\left(\lambda_{p}=364 \mathrm{~nm}\right)$ [29]. The parametric source, which has been described in detail in previous papers [25, 29], is schematically shown in Fig. 1a. Here we remember that the polarization entangled states $|\Phi\rangle=\frac{1}{\sqrt{2}}\left(|H\rangle_{u}|H\rangle_{d}+e^{i \theta}|V\rangle_{u}|V\rangle_{d}\right)$ are obtained by superposition of the two overlapping radiation cones of the crystal corresponding to the degenerate wavelength, $\lambda=728 \mathrm{~nm}$. The phase $\theta$ of the state is controlled by micrometric displacements of the mirror $M$ (see caption of Fig. 1).

Momentum entanglement is realized for either one of the two radiation cones by selecting two pairs of correlated k-modes, $l_{u}-r_{d}$ and $r_{u}-l_{d}$ within the $e-$ ring (Fig. 1a). Because of the "phase-preserving" character of the parametric process, the relative phase between the two pair emissions is set to the value $\phi=0$. Hence, for each cone, the k-entangled state $|\psi\rangle=\frac{1}{\sqrt{2}}\left(|l\rangle_{u}|r\rangle_{d}+|r\rangle_{u}|l\rangle_{d}\right)$ can be generated. In the experiment, Bob and Alice's sites are chosen in order to perform the measurements by the upper $(u)$ and lower $(d)$ detectors, respectively (cfr. Fig. 1b). The mode sets $l_{u}-l_{d}$ and $r_{u}-r_{d}$ are spatially matched in two different points of a symmetric beamsplitter $(B S)$ : Fig. 1b. A trombone mirror assembly mounted on a motorized translation stage (not shown in the Figure) allows fine adjustments of the path delay $\Delta x$ between the input modes $l_{u}-l_{d}$ and $r_{u}-r_{d}$. The photons associated with the output $B S$ modes, $l_{u}^{\prime}-l_{d}^{\prime}$ and $r_{u}^{\prime}-r_{d}^{\prime}$, are detected by four single photon detectors (Fig. 1b) within a bandwidth $\Delta \lambda=6 \mathrm{~nm}$ which corresponds

\begin{tabular}{c|cccc}
\hline \hline & $A_{\mathbf{k}} B_{\mathbf{k}}$ & $A_{\mathbf{k}} b_{\mathbf{k}}$ & $a_{\mathbf{k}} B_{\mathbf{k}}$ & $a_{\mathbf{k}} b_{\mathbf{k}}$ \\
\hline $\mathbf{A}_{\pi} \mathbf{A}_{\pi}$ & 0.9077 & 0.9097 & 0.9054 & 0.9133 \\
$\mathbf{a}_{\pi} \mathbf{a}_{\pi}$ & -0.8994 & -0.9128 & -0.8962 & -0.9069 \\
$\mathbf{B}_{\pi} \mathbf{b}_{\pi}$ & -0.8955 & -0.9033 & -0.9052 & -0.9008 \\
$\mathbf{b}_{\pi} \mathbf{B}_{\pi}$ & -0.9143 & -0.9206 & -0.9181 & -0.9196 \\
\hline \hline
\end{tabular}

\begin{tabular}{c|cccc}
\hline \hline & $\mathbf{A}_{\mathbf{k}} \mathbf{A}_{\mathbf{k}}$ & $\mathbf{a}_{\mathbf{k}} \mathbf{a}_{\mathbf{k}}$ & $\mathbf{B}_{\mathbf{k}} \mathbf{B}_{\mathbf{k}}$ & $\mathbf{b}_{\mathbf{k}} \mathbf{b}_{\mathbf{k}}$ \\
\hline$A_{\pi} B_{\pi}$ & 0.8920 & 0.8330 & 0.8355 & 0.8682 \\
$A_{\pi} b_{\pi}$ & 0.8954 & 0.8312 & 0.8289 & 0.8550 \\
$a_{\pi} B_{\pi}$ & 0.8970 & 0.8313 & 0.8304 & 0.8632 \\
$a_{\pi} b_{\pi}$ & 0.8837 & 0.8267 & 0.8330 & 0.8590 \\
\hline \hline
\end{tabular}

TABLE I: Above: experimental values of the polarization observables $A_{\pi} A_{\pi}, a_{\pi} a_{\pi}, B_{\pi} b_{\pi}$, and $b_{\pi} B_{\pi}$ (bold) measured for different settings of the momentum observables $A_{\mathbf{k}} B_{\mathbf{k}}, A_{\mathbf{k}} b_{\mathbf{k}}$, $a_{\mathbf{k}} B_{\mathbf{k}}$, and $a_{\mathbf{k}} b_{\mathbf{k}}$ (italics); below: experimental values of the momentum observables $A_{\mathbf{k}} A_{\mathbf{k}}, a_{\mathbf{k}} a_{\mathbf{k}}, B_{\mathbf{k}} B_{\mathbf{k}}$, and $b_{\mathbf{k}} b_{\mathbf{k}}$ (bold) measured for different settings of the polarization observables $A_{\pi} B_{\pi}, A_{\pi} b_{\pi}, a_{\pi} B_{\pi}$, and $a_{\pi} b_{\pi}$ (italics). Experimental uncertainties are typically of the order of 0.0020 .

to a coherence-time of the down converted photons: $\tau_{c o h}$ $\approx 150 \mathrm{fsec}$. Polarization analysis could be performed by using a half-wave plate and a polarizing beamsplitter in each detection arm. By varying the path delay around $\Delta x=0$, we could observe a dip in the two-photon coincidences for the mode combinations $l_{u}^{\prime}-r_{d}^{\prime}$ and $r_{u}^{\prime}-l_{d}^{\prime}$, while a peak was observed in both cases: $l_{u}^{\prime}-l_{d}^{\prime}, r_{u}^{\prime}-r_{d}^{\prime}$ 25]. The measured resonance "visibility" $(v \approx 0.90)$ was high enough to obtain a violation of the Bell inequality (71).

Let us describe the different measurements performed in our experiment. Every experimental result we show corresponds to a measurement lasting an average time of 10 sec. As a preliminary step, we verified the violation of Bell inequalities on each degree of freedom by separate measurements performed on polarization and linear momentum by using the state (11). The corresponding experimental results are: $\left|\left\langle\beta_{\pi}\right\rangle\right|=2.5762 \pm 0.0068$ and $\left|\left\langle\beta_{\mathbf{k}}\right\rangle\right|=2.5658 \pm 0.0067$ with a violation of local realism by 85 and 84 standard deviations, respectively.

Before demonstrating the exponential growth of the Bell inequality violation with $N=2$ of degrees of freedom of a $n=2$-photon entangled state, we verified the preliminary theoretical assumptions $(i)$ and $(i i)$. Specifically, we performed the measurement of the operators $A_{\pi} A_{\pi}, a_{\pi} a_{\pi}, B_{\pi} b_{\pi}$, and $b_{\pi} B_{\pi}$, where the first (second) operator refers to the $u(d)$ side. In order to verify $(i i)$, each measurement has been performed for any setting of momentum analysis involved in the experiment, namely $A_{\mathbf{k}} B_{\mathbf{k}}, A_{\mathbf{k}} b_{\mathbf{k}}, a_{\mathbf{k}} B_{\mathbf{k}}$, and $a_{\mathbf{k}} b_{\mathbf{k}}$. The experimental results are shown in Table I (above). An analogous procedure was followed for the momentum observables $A_{\mathbf{k}} A_{\mathbf{k}}, a_{\mathbf{k}} a_{\mathbf{k}}$, $B_{\mathbf{k}} b_{\mathbf{k}}$, and $b_{\mathbf{k}} B_{\mathbf{k}}$ [Table I (below)]. The results in both Tables support both assumptions with a reasonably high 


\begin{tabular}{c|cccc}
\hline \hline & $\mathbf{A}_{\mathbf{k}} \mathbf{B}_{\mathbf{k}}$ & $\mathbf{A}_{\mathbf{k}} \mathbf{b}_{\mathbf{k}}$ & $\mathbf{a}_{\mathbf{k}} \mathbf{B}_{\mathbf{k}}$ & $\mathbf{a}_{\mathbf{k}} \mathbf{b}_{\mathbf{k}}$ \\
\hline $\mathbf{A}_{\pi} \mathbf{B}_{\pi}$ & 0.4210 & -0.4826 & 0.5080 & 0.5108 \\
$\mathbf{a}_{\pi} \mathbf{B}_{\pi}$ & -0.5010 & 0.4490 & -0.3254 & -0.3792 \\
$\mathbf{A}_{\pi} \mathbf{b}_{\pi}$ & -0.4801 & 0.3985 & -0.3205 & -0.4431 \\
$\mathbf{a}_{\pi} \mathbf{b}_{\pi}$ & -0.4508 & 0.4536 & -0.4479 & -0.4475 \\
\hline \hline
\end{tabular}

TABLE II: Experimental values of the 16 different joint measurements of the polarization, $A_{\pi} B_{\pi}, A_{\pi} b_{\pi}, a_{\pi} B_{\pi}$, and $a_{\pi} b_{\pi}$, and the momentum observables $A_{\mathbf{k}} B_{\mathbf{k}}, A_{\mathbf{k}} b_{\mathbf{k}}, a_{\mathbf{k}} B_{\mathbf{k}}$, and $a_{\mathbf{k}} b_{\mathbf{k}}$ performed on both photons. Experimental uncertainties are typically of the order of 0.0040 . The exact values will be reported shortly in a more extended paper.

degree of certainty. The violation of the Bell inequality (7) was demonstrated by performing 16 different simultaneous measurements of the polarization observables $A_{\pi} B_{\pi}, A_{\pi} b_{\pi}, a_{\pi} B_{\pi}$, and $a_{\pi} b_{\pi}$, and the momentum observables $A_{\mathbf{k}} B_{\mathbf{k}}, A_{\mathbf{k}} b_{\mathbf{k}}, a_{\mathbf{k}} B_{\mathbf{k}}$, and $a_{\mathbf{k}} b_{\mathbf{k}}$ on both photons. The probabilities of each outcome for the $16 \pi-\vec{k}$ settings are summarized in Table II.

The experimental value of $|\beta|$, obtained after summation over all the measured values of Table II, $|\langle\beta\rangle|=$ $7.019 \pm 0.015$, corresponds to a violation of the inequality (7) by 196 standard deviations, demonstrating the magnitude of the contradiction with local realism achievable with the 2-photon hyper-entangled state (1). Assuming the version of EPR elements of reality proposed in [28], we have obtained an experimental value of $|\langle\beta\rangle| / 4=$ 1.7548 for two degrees of freedom (polarization and path) vs an experimental value of $\left|\left\langle\beta_{\pi}\right\rangle\right| / 2=1.2881$ for polarization, and $\left|\left\langle\beta_{\mathbf{k}}\right\rangle\right| / 2=1.2829$ for path.

In this Letter we have given the first experimental demonstration of Mermin's prediction that the nonlocal character of a quantum state grows with the dimension of the Hilbert space 9]. The experiment has been performed by using a polarization momentum two-photon hyper-entangled state. A further extension to a larger Hilbert space could show an even more significant deviation from classical bounds by entangling both particles in other degrees of freedom of the hyper-entangled state. We are presently investigating in our Laboratory the adoption of time bin for this purpose.

This work was supported by the FIRB 2001 and PRIN 2005 of MIUR (Italy) and by the FET European Network on Quantum Information and Communication, and the Spanish MEC Project No. FIS2005-07689.

[1] A. Einstein, B. Podolsky, and N. Rosen, Phys. Rev. 47, 777 (1935).
[2] J. S. Bell, Physics (Long Island City, NY) 1, 195 (1964).

[3] N. D. Mermin, Phys. Rev. D 22, 356 (1980).

[4] A. Garg and N. D. Mermin, Phys. Rev. Lett. 49, 901 (1982); 49, 1294 (1982).

[5] A. Garg and N. D. Mermin, Phys. Rev. D 27, 339 (1983).

[6] N. Gisin and A. Peres, Phys. Lett. A 162, 15 (1992).

[7] D. G. Collins, N. Gisin, N. Linden, S. Massar, S. Popescu, Phys. Rev. Lett. 88, 040404 (2002).

[8] J.-L. Chen, D. Kaszlikowski, L. C. Kwek, C. H. Oh, and M. Żukowski, Phys. Rev. A 64, 052109 (2001).

[9] N. D. Mermin, Phys. Rev. Lett. 65, 1838 (1990).

[10] D. M. Greenberger, M. A. Horne, and A. Zeilinger, in Bell's Theorem, Quantum Theory, and Conceptions of the Universe, edited by M. Kafatos (Kluwer, Dordrecht, 1989), p. 69.

[11] D. Bouwmeester, J.-W. Pan, M. Daniell, H. Weinfurter, and A. Zeilinger, Phys. Rev. Lett. 82, 1345 (1999).

[12] J.-W. Pan, D. Bouwmeester, M. Daniell, H. Weinfurter, and A. Zeilinger, Nature (London) 403, 515 (2000).

[13] J.-W. Pan, M. Daniell, S. Gasparoni, G. Weihs, and A. Zeilinger, Phys. Rev. Lett. 86, 4435 (2001).

[14] Z. Zhao, T. Yang, Y.-A. Chen, A.-N. Zhang, M. Żukowski, J.-W. Pan, Phys. Rev. Lett. 91, 180401 (2003).

[15] M. Hein, W. DÄur, and H.-J. Briegel, Phys. Rev. A 71, 032350 (2005).

[16] A. Cabello, Phys. Rev. Lett. 87, 010403 (2001).

[17] P. K. Aravind, Found. Phys. Lett. 15, 397 (2002).

[18] A. Cabello, Phys. Rev. Lett. 95, 210401 (2005).

[19] A. Cabello, Phys. Rev. A 72, 050101(R) (2005).

[20] C. Cinelli, M. Barbieri, R. Perris, P. Mataloni, and F. De Martini, Phys. Rev. Lett. 95, 240405 (2005).

[21] T. Yang, Q. Zhang, J. Zhang, J. Yin, Z. Zhao, M. Żukowski, Z.-B. Chen, and J.-W. Pan, Phys. Rev. Lett. 95, 240406 (2005).

[22] A. Cabello, "Bell Inequalities Based on Equalities" (work in progress).

[23] P. G. Kwiat, J. Mod. Opt. 44, 2173 (1997).

[24] P. G. Kwiat and H. Weinfurter, Phys. Rev. A 58, R2623 (1998).

[25] M. Barbieri, C. Cinelli, F. De Martini, and P. Mataloni, Phys. Rev. A 72, 052110 (2005).

[26] J. T. Barreiro, N. K. Langford, N. A. Peters, and P. G. Kwiat, Phys. Rev. Lett. 95, 260501 (2005).

[27] J. F. Clauser, M. A. Horne, A. Shimony, and R. A. Holt, Phys. Rev. Lett. 23, 880 (1969).

[28] P. H. Eberhard and P. Rosselet, Found. Phys. 25, 91 (1995).

[29] C. Cinelli, G. Di Nepi, F. De Martini, M. Barbieri, and P. Mataloni, Phys. Rev. A 70, 022321 (2004). 